\title{
Pseudo-Schwarzschild description of accretion-powered spherical outflow
}

\author{
Tapas K. Das \\ Inter University Centre for Astronomy and Astrophysics Post Bag 4 Ganeshkhind Pune 411007 India \\ Email:tapas@iucaa.ernet.in
}

Accepted ....... Received ........ ; in original form ........

\begin{abstract}
Using two different pseudo-Schwarzschild potentials proposed by Artemova et. al (1996), we formulate and solve the equations governing spherically symmetric transonic inflow and outflow in presence of a relativistic hadronic pressure mediated steady, standing, spherical shock around the central compact object and then we selfconsistently connect the accretion-wind solutions to calculate the mass outflow rate $R_{\dot{m}}$ in terms of minimum number of flow parameters. Also we study the dependence of this rate on various boundary conditions governing the flow.
\end{abstract}

Key words: accretion, accretion discs - black hole physics - outflow - wind - hydrodynamics

\section{INTRODUCTION}

For spherically symmetric accretion onto a non-rotating compact object, a novel mechanism was proposed (Protheros \& Kazanas 1983, Kazanas \& Ellison 1986, hereafter PK83 and KE86 respectively) in which the kinetic energy of the accreting material was shown to be randomized by proposing a steady, collision-less, relativistic hadronic-pressuresupported spherical shock surface around the accreting Schwarzschild black hole which produces a non-thermal spectrum of relativistic protons. In a previous work (Das 1999, hereafter D99), we have explicitly calculated the exact location (radial distance measured from the central accretor in units of Schwarzschild radius $r_{g}=\frac{2 G M_{B H}}{c^{2}}$, where $M_{B H}$ is the mass of the black hole, $G$ is the Universal gravitational constant and $c$ is the velocity of light in vacuum) of the above mentioned shock in terms of only three accretion parameters, namely, the specific energy of the flow $\mathcal{E}$, accretion rate $\dot{M}_{E d d}$ (scaled in units of Eddington rate) and the adiabatic index $\gamma_{i n}$ of the inflow for spherically-symmetric, transonic accretion of adiabatic fluid onto a Schwarzschild black hole. By solving the set of hydrodynamic equations describing the motion of accreting material under the influence of modified Newtonian potential proposed by Paczyński \& Wiita (1980), we have shown there that it is possible to construct a self-consistent inflow-outflow system where a part of the accreting material may be blown as wind from the spherical shock surface and the mass outflow rate $R_{\dot{m}}$ (the measure of the fraction of accreting material being 'kicked off' as wind) was computed in terms of various accretion as well as shock parameters.

However, other than the Paczyński-Wiita potential, a number of modified Newtonian potentials of various forms are available in the literature which accurately approximate some general relativistic effects important for studying accretion discs around a Schwarzschild black hole. Such potentials may be called 'pseudo-Schwarzschild' potentials because they nicely mimic the space-time around a nonrotating/slowly rotating compact object. Recently, we have shown that (Das \& Sarkar 2001, hereafter DS) though these so called pseudo potentials were originally proposed to mimic the relativistic effects manifested in disc accretion, it is quite reasonable to use most of these potentials in studying various dynamical and thermodynamic quantities also for spherical accretion on to Schwarzschild black holes and it was established that along with the Paczyński and Wiita potential, two other potentials proposed by Artemova et. al. (1996) also provide reasonably good approximation to the complete general relativistic description of transonic, spherically symmetric accretion on to a Schwarzschild black hole.

Remembering that the free-fall acceleration plays a very crucial role in Newtonian gravity, Artemova et. al. (1996) proposed these two potentials to study disc accretion around a non-rotating black hole. The first potential proposed by them produces exactly the same value of the free-fall acceleration of a test particle at a given value of $r$ as is obtained 
for a test particle at rest with respect to the Schwarzschild reference frame, and is given by

$$
\Phi_{1}=-1+\left(1-\frac{1}{r}\right)^{\frac{1}{2}}
$$

The second one gives the value of the free fall acceleration that is equal to the value of the covariant component of the three dimensional free-fall acceleration vector of a test particle that is at rest in the Schwarzschild reference frame and is given by

$$
\Phi_{2}=\frac{1}{2} \ln \left(1-\frac{1}{r}\right)
$$

For Eq. 1(a-b), $r$ represents the radial co-ordinate scaled in the unit of $r_{g}$. Efficiencies produced by $\Phi_{1}$ and $\Phi_{2}$ are -0.081 and -0.078 respectively. As both $\Phi_{1}$ and $\Phi_{2}$ stems from the consideration of free fall acceleration and calculates the dependence of free fall acceleration on radial distance in the Schwarzschild metric (which describes a spherically symmetric gravitational field in vacuum), it appears to be quite justified to use those potentials to study spherically symmetric accretion.

Owing to the fact that $\Phi_{1}$ and $\Phi_{2}$ may be used to mimic the spacetime around a spherically accreting non-rotating black hole quite nicely, we believe that along with the calculation presented in D99, it is equally important to investigate the various features of the accretion powered spherical outflow using these two potentials. In this paper we precisely do this, first we formulate and solve the equations governing the inflow and outflow using $\Phi_{1}$ and $\Phi_{2}$ (in presence of a steady, standing spherical shock as described in previous paragraphs) and then self-consistently connect the accretion and wind solutions to calculate what fraction of the accreting material is being blown as wind. Also we study the dependence of this fraction on various flow parameters. The plan of this paper is as follows. In next section we describe how to formulate and solve the governing equations. In $\S 3$, we present our results. In $\S 4$, the results will be reviewed and conclusions will be drawn.

\section{GOVERNING EQUATIONS AND SOLUTION PROCEDURE}

\subsection{Inflow model}

Hereafter we will refer to these two potentials as $\Phi_{i}$ in general where $\{i=1,2\}$ would correspond to $\Phi_{1}$ (Eq. (1a)) and $\Phi_{2}$ (Eq. (1b)) respectively. We assume that a Schwarzschild type black hole spherically accretes fluid obeying a polytropic equation of state. The density of the fluid is $\rho(r), r$ being the radial distance measured in the unit of Schwarzschild radius $r_{g}$. We also assume that the accretion rate (scaled in the unit of Eddington rate $\dot{M}_{E d d}$ ) is not a function of $r$ and we ignore the self-gravity of the flow. For simplicity of calculation, we choose the geometric unit where the unit of length is scaled in units of $r_{g}$, units of velocity in units of $c$. All other physically relevant quantities can be expressed likewise. We also set $G=c=1$ in the system of units used here. It is to be mentioned here that one fundamental criterion for formation of hydrodynamic outflows is that the outflowing wind should have a positive Bernoulli constant which means that the matter in the post-shock region is able to escape to infinity. However, positiveness in Bernoulli's constant may lead to another situation as well where shock may quasi-periodically originate at some certain radius and propagate outwards without formation of outflows. So formation of outflows is one of the possible scenarios when we focus on the positive energy solutions. In this paper we concentrate only on solutions producing outflows, thus we use only the positive Bernoulli constant throughout our model which is standard practice for studying hydrodynamic winds. Another assumption made in this paper is to treat the accreting as well as post-shock matter as a single temperature fluid, the temperature of which is basically characterized by proton temperature.

For any pseudo-Schwarzschild potential $\Phi_{i}$, the equation of motion for spherically accreting material onto the accretor is given by

$$
\frac{\partial u}{\partial t}+u \frac{\partial u}{\partial r}+\frac{1}{\rho} \frac{\partial P}{\partial r}+\Phi_{i}^{\prime}=0
$$

where symbols have their usual meaning. The first term of Eq. 2(a) is the Eulerian time derivative of the dynamical velocity at a given $r$, the second term is the 'advective' term, the third term is the momentum deposition due to pressure gradient and the final term is due to the gravitational acceleration for a particular $i$ th potential $\Phi_{i}$. The continuity equation can be written as

$$
\frac{\partial \rho}{\partial t}+\frac{1}{r^{2}} \frac{\partial}{\partial r}\left(\rho u r^{2}\right)=0
$$

For a polytropic equation of state, i.e., $p=K \rho^{\gamma}$, the steady state solutions of eqn. 2(a) and eqn. 2(b) are

1) Conservation of specific energy $\mathcal{E}$ of the flow:

$$
\mathcal{E}=\frac{u^{2}}{2}+\frac{a^{2}}{\gamma_{\text {in }}-1}+\Phi_{i}
$$

where $\gamma_{i n}$ is the adiabatic index of the inflow (accretion) and

2) Conservation of Baryon number (or accretion rate $\dot{M}_{i n}$ ):

$$
\dot{M}_{i n}=4 \pi \rho u r^{2}
$$

Using $\dot{\mathcal{M}}_{\text {in }}$ as the entropy accretion rate where $\dot{\mathcal{M}}=$ $\dot{M}_{i n} \gamma_{i n} \frac{1}{\gamma_{i n}-1} K^{\frac{1}{\gamma_{i n}-1}}$, eqn. $3(\mathrm{~b})$ can be rewritten as (see DS and references therein):

$$
\dot{\mathcal{M}}_{\text {in }}=4 \pi a^{\frac{2}{\gamma_{\text {in }}-1}} u r^{2}
$$

It is now quite straightforward to derive the space gradient of dynamical velocity $\left(\frac{d u}{d r}\right)_{i}$ for flow in any particular $i$ th potential $\Phi_{i}$ as

$$
\left(\frac{d u}{d r}\right)_{i}=\frac{\frac{2 a^{2}}{r}-\Phi_{i}{ }^{\prime}}{u-\frac{a^{2}}{u}}
$$

where $\left|\Phi^{\prime}{ }_{i}\right|$ denotes the absolute value of the space derivative of $\Phi_{i}$, i.e.,

$$
\left|\Phi^{\prime}{ }_{i}\right|=\left|\frac{d \Phi_{i}}{d r}\right|
$$

Since the flow is assumed to be smooth everywhere, if the denominator of Eq. 4(a) vanishes at any radial distance $r$, the numerator must also vanish there to maintain the continuity of the flow. One therefore arrives at the so called 'sonic 
point conditions' by simultaneously making numerator and denominator of Eq. 4(a) equal to zero and the sonic point conditions can be expressed as follows

$$
u_{c}^{i}=a_{c}^{i}=\sqrt{\left.\frac{r_{c}^{i}}{2} \Phi_{i}{ }^{\prime}\right|_{c}}
$$

where superscript $i$ denotes the specific value of sonic quantities for a particular $i$ th potential $\Phi_{i}$, and $\left.\Phi_{i}^{\prime}\right|_{c}$ is the value of $\left(\frac{d \Phi_{i}}{d r}\right)$ evaluated at the corresponding sonic point $r_{c}^{i}$. The value of sonic point $r_{c}^{i}$ for any $i$ th potential $\Phi_{i}$ can be obtained by algebraically solving the following equation

$$
\mathcal{E}-\left.\frac{1}{2}\left(\frac{\gamma_{i n}+1}{\gamma_{i n}-1}\right) r_{c}^{i} \Phi_{i}{ }^{\prime}\right|_{c}-\left.\Phi_{i}\right|_{c}=0
$$

where $\left.\Phi_{i}\right|_{c}$ is the value of $i$ th potential at the corresponding sonic point $r_{c}^{i}$. Similarly, the value of $\left(\frac{d u}{d r}\right)_{i}$ for any $\Phi_{i}$ at its corresponding sonic point $r_{c}^{i}$ can be obtained by solving the following quadratic equation:

$$
\begin{gathered}
\left(1+\gamma_{i n}\right)\left(\frac{d u}{d r}\right)_{c, i}^{2}+2.829\left(\gamma_{i n}-1\right) \sqrt{\frac{\left.\Phi_{i}{ }^{\prime}\right|_{c}}{r_{c}^{i}}\left(\frac{d u}{d r}\right)_{c, i}} \\
+\left(2 \gamma_{i n}-1\right) \frac{\left.\Phi_{i}{ }^{\prime}\right|_{c}}{r_{c}^{i}}+\left.\Phi_{i}{ }^{\prime \prime}\right|_{c}=0
\end{gathered}
$$

where $\left.\Phi_{i}{ }^{\prime \prime}\right|_{c}$ is the value of $\frac{d^{2} \Phi_{i}}{d r^{2}}$ at the corresponding critical point $r_{c}^{i}$.

One can simultaneously solve eqn. 3(a) and eqn. 3(b) (alternatively, eqn. 3(a) and eqn. 3(c)) for any specific $\Phi_{i}$ for a fixed value of $\mathcal{E}$ and $\gamma_{\text {in }}$ to obtain various dynamical and thermodynamic flow quantities. In this work we normally use the value of $\gamma_{i n}$ to be $\frac{4}{3}$. Though far away from the black hole, optically thin accreting plasma may not be treated as ultra-relativistic fluid (by the term 'ultra-relativistic' and 'purely non-relativistic' we mean a flow with $\gamma=\frac{4}{3}$ and $\gamma=\frac{5}{3}$ respectively, according to the terminology used in Frank et. al. 1992) in general, close to the hole it always advects with enormously large radial velocity and could be well-approximated as ultra-relativistic flow. As because our main region of interest, the shock formation zone, normally lies close to the black hole (a few tens of $r_{g}$ away from the hole or sometimes even less, see results and figures in $\S 3$ ), we believe that it is fairly justifiable to assign the value $\frac{4}{3}$ for $\gamma_{i n}$ in our work. However, to rigorously model a real flow without any assumption, a variable polytropic index having proper functional dependence on radial distance (i.e., $\left.\gamma_{i n} \equiv \gamma_{i n}(r)\right)$ might be considered instead of using a constant $\gamma_{i n}$, and equations of motion might be formulated accordingly, which we did not attempt here for the sake of simplicity. Nevertheless, we keep our option open for values of $\gamma_{\text {in }}$ other than $\frac{4}{3}$ as well and investigated the outflow for an range of values of $\gamma_{i n}$ for a specific value of $\mathcal{E}$ and $\dot{M}_{E d d}$ (Fig. $4, \S 3.3$ ). The same kind of investigations could be performed for a variety of values of $\mathcal{E}$ and $\dot{M}_{E d d}$ and a set of results may be obtained with a wide range of values of $\gamma_{i n}$ which tells that our calculation is not restricted to the value $\gamma_{i n}=\frac{4}{3}$ only; rather the model is general enough to deal with all possible value of $\gamma_{i n}$ for polytropic accretion.

\subsection{Shock parameters and the outflow model}

As already mentioned, a steady, collision-less shock forms due to the instabilities in the plasma flow (see D99 and references therein for details of shock formation mechanism). We take this shock surface to be the effective physical barrier around the black hole which might be responsible to generate the outflow. We assume that the presence of a collisionless steady standing spherical shock discussed in this work may randomize the directed in-fall motion and at the shock surface the individual components of the total energy of the flow (which is a combination of the kinetic, thermal and gravitational energy) gets rearranged in such a manner that the thermal energy of the post-shock matter dominates (due to enormous shock generated post-shock proton temperature) over the gravitational attraction of the accretor and a part of the in-falling material is driven by thermal pressure to escape to infinity as wind. In $\S 3$. we show that for any shock solution, the mass-loss rate normally co-relates with the post-shock proton temperature which essentially supports the validity of our assumption. We also assume that for our model, the effective thickness of the shock $\Delta_{s h}$ is small enough compared to the shock standoff distance $r_{s h}$, and that the relativistic particles encounter a full shock compression ratio while crossing the shock.

At the shock, density of matter will shoot up and inflow velocity will drop abruptly. If $\left(\rho_{-}, u_{-}\right)$and $\left(\rho_{+}, u_{+}\right)$are the pre- and post-shock densities and velocities respectively at the shock surface, then

$$
\frac{\rho_{+}}{\rho_{-}}=R_{c o m p}=\frac{u_{-}}{u_{+}}
$$

where $R_{\text {comp }}$ is the shock compression ratio. For high shock Mach number solution, the expression for $R_{\text {comp }}$ can be well approximated as

$$
R_{\text {comp }}=1.44 M_{s h}{ }^{\frac{3}{4}}
$$

where $M_{s h}$ is the shock Mach number and Eq. (5) holds for $M_{s h} \gtrsim 4.0$ (Ellison \& Eichler 1985).

In terms of various accretion parameters, shock location can be computed as (D99):

$$
r_{s h}=\frac{3 \sigma_{p p} \dot{M}_{E d d}}{4 \pi u_{s h}^{2}}\left(\frac{1-2.4 M_{s h}{ }^{-0.68}}{1-3.2 M_{s h}^{-0.62}}\right)
$$

where $\sigma_{p p}$ is the collision cross section for relativistic protons, $u_{s h}$ and $M_{s h}$ are the dynamical flow velocity and the Mach number attained at the shock location, $\dot{M}_{E d d}$ is the mass accretion rate scaled in units of Eddington rate. One can understand that

$$
\left(u_{s h}, M_{s h}\right) \equiv \zeta\left(\mathcal{E}, \dot{M}_{E d d}, \gamma_{i n}\right)
$$

where $\zeta$ has some complicated non-linear functional form which cannot be evaluated analytically, but the value of $u_{s h}$ 
and $M_{s h}$ can easily be obtained in terms of $\left\{\mathcal{E}, \dot{M}_{E d d}, \gamma_{i n}\right\}$ by numerically solving Eq. 3(a-b) and Eq. 7 (with the help of Eq. 4(a-d)) simultaneously. Hence one can write

$$
r_{s h} \equiv \xi\left(\mathcal{E}, \dot{M}_{E d d}, \gamma_{i n}\right)
$$

where $\xi$ has some functional form other than that of $\zeta$. In ordinary stellar mass-loss computations (Tarafder 1988, and references therein), the outflow is assumed to be isothermal till the sonic point. This assumption is probably justified, since copious photons from the stellar atmosphere deposit momenta on the slowly outgoing and expanding outflow and possibly make the flow close to isothermal. This need not be the case for outflow from black hole candidates. Our effective boundary layer, being close to the black hole, are very hot and most of the photons emitted may be swallowed by the black hole itself instead of coming out of the region and depositing momentum onto the outflow. Thus, the outflow could be cooler than the isothermal flow in our case. We choose polytropic outflow with a different polytropic index $\gamma_{o}<\gamma_{i n}$ due to momentum deposition. In our calculation we also assume that essentially the post-shock fluid pressure and the post-shock proton temperature controls the wind formation as well as the barionic matter content of the outflow.

The adiabatic post-shock sound speed $a_{s h}^{+}$and the postshock temperature $T_{s h}^{+}$(which is basically the temperature of the protons according to our one-temperature fluid approximation) can be calculated as:

$$
a_{s h}^{+}=\sqrt{\frac{\gamma_{o} p_{s h}^{+}}{\rho_{s h}^{+}}}
$$

and

$$
T_{s h}^{+}=\frac{\mu m_{p} p_{s h}^{+}}{\kappa \rho_{s h}^{+}}
$$

where $p_{s h}^{+}$and $\rho_{s h}^{+}$are the post-shock pressure and density of the flow at shock location $r_{s h}$ respectively. For low energy accretion ('cold' inflow, so to say) which is appropriate to produce a high shock Mach number solution, one can assume that the pre-shock thermal pressure $\left(p_{s h}^{-}\right)$may be neglected compared to its post-shock value $\left(p_{s h}^{+}\right)$and to the pre-shock ram pressure $\left(\rho_{s h}^{-}\left(u_{s h}^{-}\right)^{2}\right)$. One can obtain the value of $p_{s h}^{+}$ using Eq. (5-6) and from the total pressure balance condition at shock as,

$$
p_{s h}^{+}=\left(u_{s h}^{+}\right)^{2} r_{s h}\left(\frac{R_{c o m p}-1}{R_{c o m p}}\right)
$$

Combining Eq. (5-9), post-shock sound velocity and temperature obtained at the shock surface can be rewritten as:

$$
a_{s h}^{+}=3.54\left(r_{s h} u_{s h}^{+}\right)^{1.5} \sqrt{\frac{\gamma_{o}}{\dot{M}_{E d d}}\left(\frac{1.44 M_{s h}^{\frac{3}{4}}-1}{M_{s h}^{\frac{3}{4}}}\right)}
$$

and

$$
T_{s h}^{+}=\frac{4 \pi \mu m_{p}}{\kappa \dot{M}_{E d d}}\left(r_{s h} u_{s h}^{+}\right)^{3}\left(\frac{1.44 M_{s h}^{\frac{3}{4}}-1}{M_{s h}^{\frac{3}{4}}}\right)
$$

General form of the conservation equations governing the polytropic outflow will be the same as Eq. (3a - 3c) with different polytropic index and total specific energy and entropy $\left(\mathcal{E}>\mathcal{E}^{\prime}, \dot{\mathcal{M}}_{\text {in }}<\dot{\mathcal{M}}^{\prime}\right.$ and $\gamma_{i n}>\gamma_{o}$, see D99). So we can write:

$$
\begin{gathered}
\mathcal{E}^{\prime}=\frac{\left(u^{o}\right)^{2}}{2}+\frac{\left(a^{o}\right)^{2}}{\gamma_{o}-1}+\Phi_{i} \\
\dot{M}_{\text {out }}=4 \pi \rho^{o} u^{o} r^{2}
\end{gathered}
$$

and

$$
\dot{\mathcal{M}}^{\prime}=4 \pi\left(a^{o}\right)^{\frac{2}{\gamma_{o}-1}} u^{o} r^{2}
$$

where where $\mathcal{E}^{\prime}$ is the specific energy of the outflow which is also assumed to be constant throughout the flow and $\dot{\mathcal{M}}^{\prime}=\dot{M}_{\text {out }} \gamma_{o} \frac{1}{\gamma_{o}-1} K^{o \frac{1}{\gamma_{o}-1}}$ is the entropy accretion rate of the outflow. $\gamma_{o}<\gamma_{i n}$ as already mentioned. Any sub/ super-script indicates that the quantities are measured for the outflow. Like Eqs. (4a - 4d), one can easily write the sonic point conditions and the velocity gradient of the outflow as:

$$
\begin{gathered}
\left(\frac{d u^{o}}{d r}\right)_{i}=\frac{\frac{2\left(a^{o}\right)^{2}}{r}-\Phi_{i}{ }^{\prime}}{u^{o}-\frac{\left(a^{o}\right)^{2}}{u^{o}}} \\
\left(u_{c}^{i}\right)^{o}=\left(a_{c}^{i}\right)^{o}=\sqrt{\left.\frac{\left(r_{c}^{i}\right)^{o}}{2} \Phi_{i}{ }^{\prime}\right|_{c}} \\
\mathcal{E}^{\prime}-\left.\frac{1}{2}\left(\frac{\gamma_{o}+1}{\gamma_{o}-1}\right)\left(r_{c}^{i}\right)^{o} \Phi_{i}\right|_{c}-\left.\Phi_{i}\right|_{c}=0
\end{gathered}
$$

and

$$
\begin{gathered}
\left(1+\gamma_{o}\right)\left(\frac{d u^{o}}{d r}\right)_{c, i}^{2}+2.829\left(\gamma_{o}-1\right) \sqrt{\frac{\left.\Phi_{i}{ }^{\prime}\right|_{c}}{\left(r_{c}^{i}\right)^{o}}\left(\frac{d u^{o}}{d r}\right)_{c, i}} \\
+\left(2 \gamma_{o}-1\right) \frac{\left.\Phi_{i}{ }^{\prime}\right|_{c}}{\left(r_{c}{ }^{i}\right)}+\left.\Phi_{i}{ }^{\prime \prime}\right|_{c}=0
\end{gathered}
$$

We then define the mass outflow rate $R_{\dot{m}}$ as:

$$
R_{\dot{m}}=\frac{\dot{M}_{\text {out }}}{\dot{M}_{\text {in }}}
$$

It is obvious from the above discussion that $R_{\dot{m}}$ should have some complicated non-linear functional dependence on the following accretion and shock parameters:

$$
R_{\dot{m}} \equiv \Psi\left(\mathcal{E}, \dot{M}_{E d d}, r_{s h}, M_{s h}, R_{c o m p}, \gamma_{i n}, \gamma_{o}\right)
$$

As $r_{s h}, M_{s h}$ and $R_{c o m p}$ can be found in terms of $\mathcal{E}, \dot{M}_{E d d}$ and $\gamma_{\text {in }}$ only, ultimately it turns out that:

$$
R_{\dot{m}} \equiv \boldsymbol{\Omega}\left(\mathcal{E}, \dot{M}_{E d d}, \gamma_{i n}, \gamma_{o}\right)
$$

Where $\boldsymbol{\Omega}$ has some complicated functional form which cannot be evaluated analytically. 


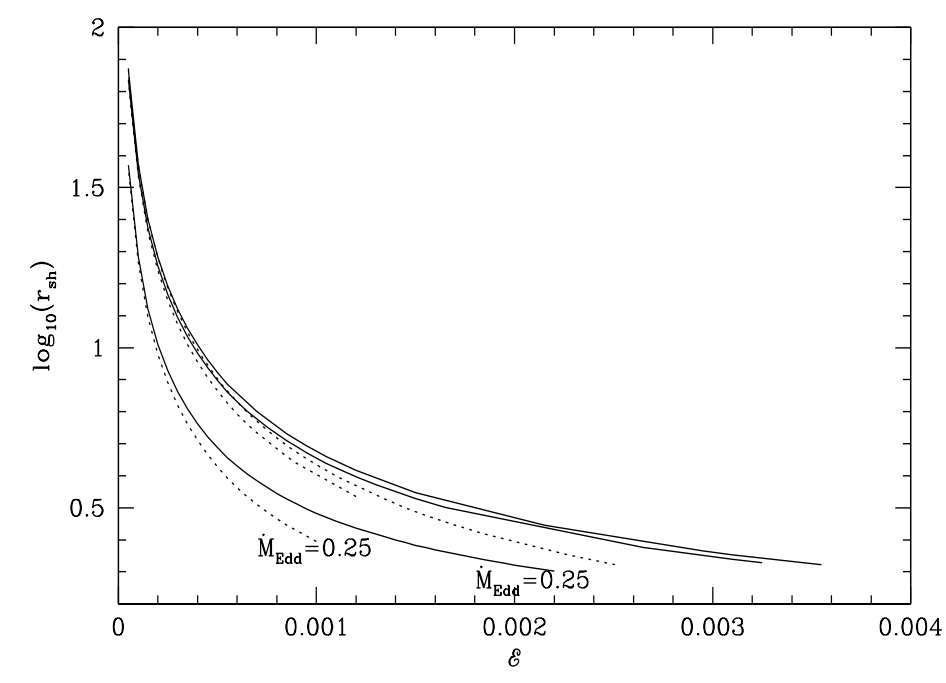

Fig. 1: Variation of shock location $r_{s h}$ (plotted in logarithmic unit along $\mathrm{Y}$ axis) with the specific energy of the flow $\mathcal{E}$ (plotted along $\mathrm{X}$ axis) for three values of accretion rate (scaled in the unit of Eddington rate $\dot{M}_{E d d}$ ) $0.25,1.0$ and 1.75 respectively from bottom to top. While the dependence for flows in $\Phi_{1}$ is shown by dotted lines, flows in $\Phi_{2}$ is shown by solid lines; see text for details.

\subsection{Simultaneous solution of inflow-outflow equations}

In this work, we are interested in finding the ratio of $\dot{M}_{\text {out }}$ to $\dot{M}_{\text {in }}$ (Eq. (13)), and not the explicit value of $\dot{M}_{\text {out }}$. Also note that the primary goal of our present work was to compute the outflow rate and to investigate its dependence on various inflow parameters but not to study the collimation procedure of the outflow.

Before we proceed in detail, a general understanding of the transonic inflow outflow system in the present case is essential to understand the basic scheme of the solution procedure. Let us consider the transonic accretion first. Infalling matter becomes supersonic after crossing a saddletype sonic point, the location of which is determined by $\left\{\mathcal{E}, \dot{M}_{E d d}, \gamma_{i n}\right\}$. This supersonic flow then encounters a shock (if present), location of which $\left(r_{s h}\right)$ is determined from Eq. (7). At the shock surface, part of the incoming matter, having a higher entropy density (because shock in a fluid flow generates entropy), is likely to return as wind through a sonic point other than the point through which it just entered. Thus a combination of transonic topologies, one for the inflow and other for the outflow (passing through a different sonic point and following topology completely different that of the 'self-wind' of the accretion), is required to obtain a full solution. So it turns out that finding a complete set of self-consistent inflow outflow solutions ultimately boils down to locate the sonic point of the polytropic outflow and the mass flux through it. Thus a supply of parameters $\mathcal{E}, \dot{M}_{E d d}, \gamma_{i n}$ and $\gamma_{o}$ make a self-consistent computation of $R_{\dot{m}}$ possible. Here $\gamma_{o}$ is supplied as free parameter because the self-consistent computation of $\gamma_{o}$ directly using $\mathcal{E}, \dot{M}_{E d d}$ and $\gamma_{i n}$ has not been attempted in this work; instead we put a constrain that $\gamma_{o}<\gamma_{i n}$ always and for any value of $\gamma_{i n}$. In reality, $\gamma_{o}$ is directly related to the heating and cooling processes taking place in the outflow.

We obtain the inflow sonic point $r_{c}$ by solving Eq. (4c). Using the fourth order the Runge Kutta method, $u(r), a(r)$ and the inflow Mach number $\left[\frac{u(r)}{a(r)}\right]$ are computed along the inflow from the inflow sonic point $r_{c}$ till the position where the shock forms. The shock location is calculated by solving Eq. (7). Various shock parameters (i.e., density, pressure etc at the shock surface) are then computed self-consistently. For outflow, with the known value of $\mathcal{E}^{\prime}$ and $\gamma_{o}$, it is easy to compute the location of the outflow sonic point $r_{c}^{o}$ from Eq. (12c). At the outflow sonic point, the outflow velocity $u_{c}^{o}$ and polytropic sound velocity $a_{c}^{o}$ is computed from Eq. (12b). Using Eq. (12a) and (12d), $\left(\frac{d u^{\circ}}{d r}\right)$ and $\left(\frac{d u^{\circ}}{d r}\right)_{c}$ is computed as was done for the inflow. Runge -Kutta method is then employed to integrate from the outflow sonic point $r_{c}^{o}$ towards the black hole to find out the outflow velocity $u^{o}$ and density $\rho^{o}$ at the shock location. The mass outflow rate $R_{\dot{M}}$ is then computed using Eq.(13).

\section{RESULTS}

\subsection{Shock location as a function of $\mathcal{E}$ and $\dot{M}_{E d d}$ and related post-shock quantities}

For a particular value of $\mathcal{E}, \dot{M}_{E d d}$ and $\gamma_{i n}$, the shock location (measured from the black hole in units of $r_{g}$ ) can be calculated using Eq. (7). As $u_{s h}$ and $M_{s h}$ is a function of $\mathcal{E}, \dot{M}_{E d d}$, and $\gamma_{i n}, r_{s h}$ will also change with the change of any of these accretion parameters. In figure 1 , we show the variation of $r_{s h}$ as a function of $\mathcal{E}$ for three different values of $\dot{M}_{E d d}$. While $\mathcal{E}$ is plotted along the X axis, shock location (in logarithmic scale) is plotted along the $\mathrm{Y}$ axis for a fixed value of $\gamma_{i n}\left(=\frac{4}{3}\right)$. Three different curves drawn by solid lines for $\Phi_{2}$ and dotted lines for $\Phi_{1}$, are plotted for three different values of $\dot{M}_{E d d}(=0.25,1.0,1.75)$. The lowermost curves (for each of the pseudo-potentials) corresponds to the value $\dot{M}_{E d d}=0.25$ as shown in the figure. For both $\Phi_{1}$ and $\Phi_{2}$, other two curves from bottom to top, correspond to $\dot{M}_{E d d}$ equal to 1.0 and 1.75 respectively. For any pseudo-potential one should note that different curves terminate (in the direction of increasing $\mathcal{E}$ ) at different points which indicates that shock formation is not a generic phenomena, i.e., shock does not form for any value of $\mathcal{E}, \dot{M}_{E d d}$, and $\gamma_{i n}$, rather a specific region of parameter space spanned by $\left\{\mathcal{E}, \dot{M}_{E d d}, \gamma_{i n}\right\}$ allows shock formation. Both sub- as well as super-Eddington accretion allows shock formation as shown in the figure. For any pseudo-potential, while the shock location non-linearly anticorrelates with $\mathcal{E}$ (for a fixed value of $\dot{M}_{E d d}$ and $\gamma_{i n}$ ), it correlates (non-linearly) with $\dot{M}_{E d d}$ (for a fixed value of $\mathcal{E}$ and $\gamma_{i n}$ ). The maximum value of $\mathcal{E}$ for which shock may form for a fixed value of $M_{E d d}$ and $\gamma_{i n}$, increases with increase of the accretion rate of the flow. It is observed that (figuratively not shown in the paper) the shock location also non-linearly anti-correlates with $\gamma_{i n}$ which means that for both $\Phi_{1}$ and $\Phi_{2}$, non-relativistic super-Eddington accccretion with low specific energy of the flow is a proper combination to produce the shock closest to the black hole (this result has significant importance in studying the amount of barionic load in the wind, see §3.3). Once the shock location is known, one can easily calculate any post-shock or shock related (the shock compression ratio for example) quantity 

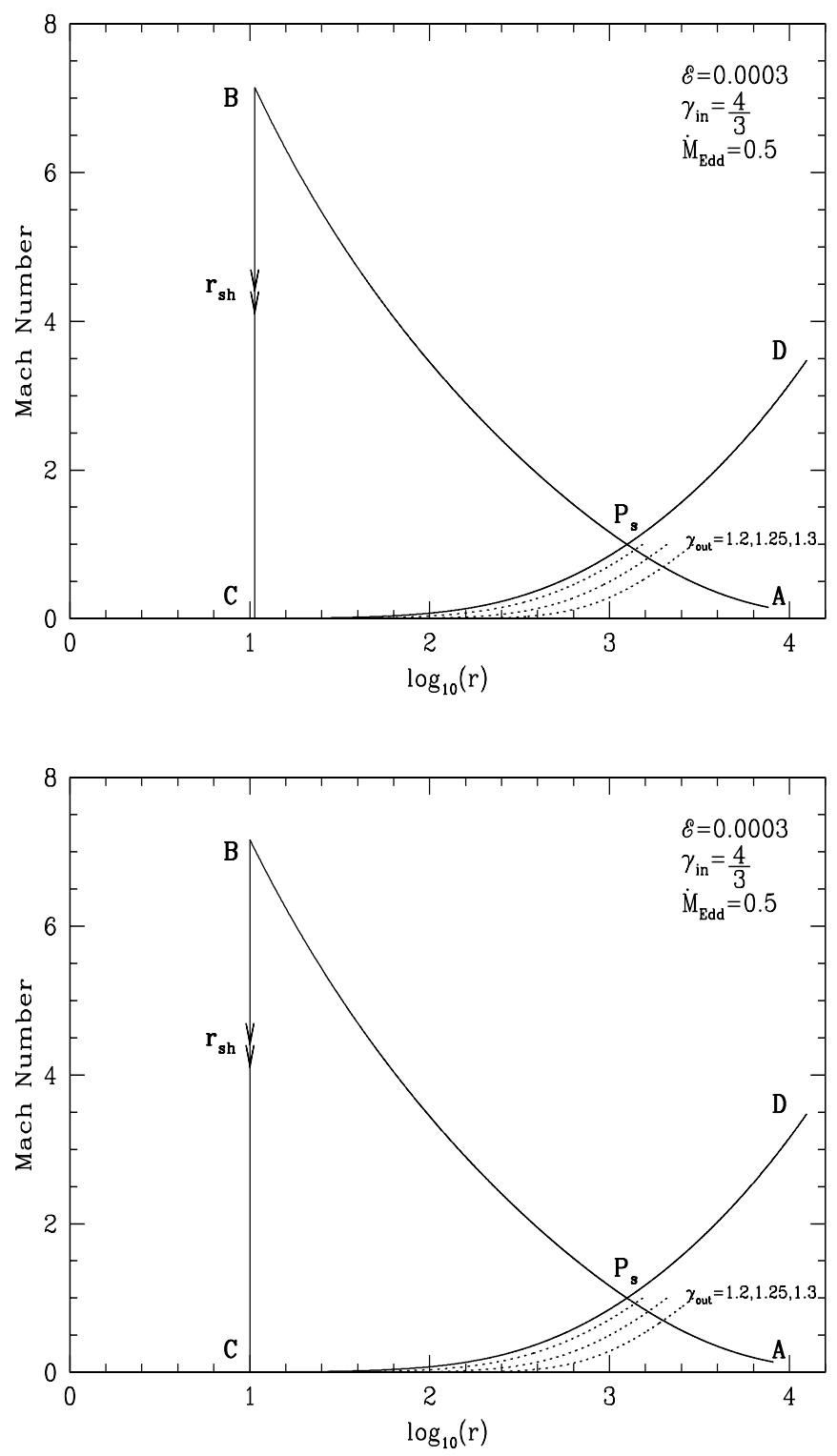

Fig. 2: Combined solution topologies for transonic accretionoutflow system for flows in $\Phi_{1}(2 \mathrm{a})$ and $\Phi_{2}(2 \mathrm{~b})$ for fixed values (shown in the figure) of accretion and outflow parameters. While Mach number is plotted along the $\mathrm{Y}$ axis, the distance (in units of $r_{g}$ ) from the event horizon of the accreting black hole is plotted along the $\mathrm{X}$ axis in logarithmic scale. Solid curve marked with $\mathrm{AB}$ represents the pre-shock transonic accretion while four dotted curves represent the accretion-powered outflow branches. Solid vertical line BC (marked by $r_{s h}$ ) with double arrow stands for the shock transition and solid line marked by $\mathrm{CD}$ stands for the 'self-wind' branch. $P_{s}$ is the location of the inflow sonic point. See text for details.

using the corresponding equations derived in $\S 2.2$. One important physical quantity of our interest is the post-shock proton temperature of the flow (which is practically the temperature of the outflow according to our one-fluid approximation, see §2.1) $T_{s h}^{+}$which can be computed using Eq. 10 (b). We have seen that for any pseudo-potential, $T_{s h}^{+}$correlates with $\mathcal{E}$ as well as with $\gamma_{i n}$, hence high energy pure- nonrelativistic flow would produce hotter outflow as well as it is evident that the closer the shock forms to the accretor, the higher becomes the post-shock proton temperature of the flow.

Whatever observations are presented in the above paragraph, is commonly applicable for both $\Phi_{1}$ and $\Phi_{2}$. However, there are a number of differences in magnitude of post-shock quantities (even in the shock location) observed when the flow is studied for two different pseudo-potentials. From figure 1 , one can observe that for the same values of $\mathcal{E}, \dot{M}_{E d d}$ and $\gamma_{i n}$, the shock forms relatively closer to the black hole for $\Phi_{2}$ compared to the case for $\Phi_{1}$. If $\left.r_{s h}\right|_{\Phi_{i}}$ is the shock location obtained using any particular $i$ th pseudo-potential, we see that:

$$
r_{s h}>\left.r_{s h}\right|_{\Phi_{1}}
$$

for a fixed value of $\left\{\mathcal{E}, \dot{M}_{E d d}, \gamma_{i n}\right\}$. This deviation is more prominent for flows with higher specific energy and the following quantity

$$
\delta r_{s h}=\left[\left.r_{s h}\right|_{\Phi_{2}}-\left.r_{s h}\right|_{\Phi_{1}}\right]
$$

decreases in the direction of low $\mathcal{E}$. Also we note that the maximum value of $\mathcal{E}$ for which the shock forms for a fixed value of $\dot{M}_{E d d}$ and $\gamma_{i n}$ (let us define that energy as $\left.\mathcal{E}_{\max }\right|_{\Phi_{i}}$ for any $i$ th pseudo-potential), is higher for flows in $\Phi_{2}$, i.e.,

$$
\left.\mathcal{E}_{\max }\right|_{\Phi_{2}}>\left.\mathcal{E}_{\max }\right|_{\Phi_{a}}
$$

but unlike $\delta r_{s h}, \delta \mathcal{E}_{\max }$ does not show any specific dependence on inflow parameters. It is also observed that (not shown in the figure) for a fixed values of energy and accretion rate, if we study the shock location as a function of the polytropic index of the inflow, the inequality presented in equation (15) is still maintained and $\delta r_{s h}$ decreases as the flow tends to its pure non-relativistic limit. As the postshock proton temperature $T_{s h}^{+}$is inversely proportional with the shock location, we find that,

$$
\left.T_{s h}^{+}\right|_{\Phi_{2}}<\left.T_{s h}^{+}\right|_{\Phi_{1}}
$$

for both the cases when

a) $\mathcal{E}$ is being varied keeping $\dot{M}_{E d d}$ and $\gamma_{i n}$ constant.

b) Flow is being studied as a function of $\gamma_{i n}$ for fixed values of $\mathcal{E}$ and $\dot{M}_{E d d}$.

However, $\delta T_{s h}^{+}$decreases with decrease in $\mathcal{E}$ but with increase in $\gamma_{i n}$.

\subsection{Combined integral curves of motion}

Figure $2 \mathrm{a}$ and $2 \mathrm{~b}$ show two typical solutions which combines the accretion and outflow for flows in $\Phi_{1}$ and $\Phi_{2}$ respectively. The accretion parameters used are $\mathcal{E}=0.0003$, 


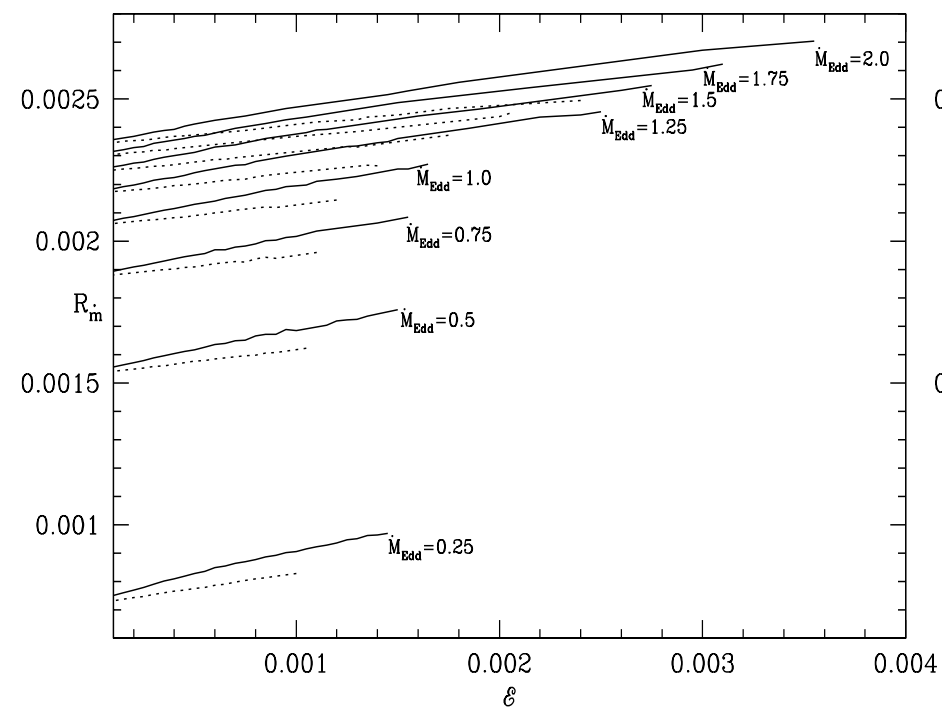

Fig. 3a:

Variation of $R_{\dot{m}}$ with inflow specific energy $\mathcal{E}$ for different values of accretion rate $\dot{M}_{E d d}$ shown in the figure. Dotted curves correspond to flow in $\Phi_{1}$ and solid curves correspond to flow in $\Phi_{2}$.

$\dot{M}_{E d d}=0.5$ and $\gamma_{i n}=\frac{4}{3}$ corresponding to ultra-relativistic inflow. For both of the figures $2 a$ and $2 b$, the solid curve $\mathrm{AB}$ represents the pre-shock region of the inflow and the solid vertical line $\mathrm{BC}$ with double arrow at $r_{s h}$ represents the shock transition. Shock locations (9.9 $r_{g}$ for the Fig. 2a and $10.6 r_{g}$ for the Fig. $2 \mathrm{~b}$ ) is obtained using the Eq.(7) for a particular set of inflow parameters mentioned above. Three dotted curves show the three different outflow branches corresponding to the three different adiabatic indices $\gamma_{o}$ of the outflow. From left to right, the values of $\gamma_{o}$ are 1.3, 1.25 and 1.2 respectively with respective mass-outflow rates as $1.558 \times 10^{-3}, 7.1487 \times 10^{-5}$ and $6.249 \times 10^{-7}$ (for Fig. 2a) and $1.588 \times 10^{-3}, 7.47 \times 10^{-5}$ and $6.765 \times 10^{-7}$ (for Fig. 2 b) respectively which indicates that for a given value of $\mathcal{E}$, $\dot{M}_{E d d}$ and $\gamma_{i n}, R_{i n}$ correlates with $\gamma_{o}$. It is evident from the figure that the outflow moves along the solution curves in a completely different way to that of the 'self-wind' solution of the inflow (solid line marked by CD in Fig. 2a and 2b). Also, the sonic points for all the outflowing branches are different to those of the accretion 'self-wind' system which is designated as $P_{s}$. While $P_{s}=1250.833 r_{g}$ for for Fig. $2 \mathrm{a}$ and $1251.665 r_{g}$ for Fig. $2 \mathrm{~b}$, the sonic points of the outflowing branches corresponding to $\gamma_{o}=1.3,1.25$ and 1.2 are $1528.55 r_{g}, 2084.033 r_{g}, 2917.31 r_{g}$ for Fig. 2a and $1529.32 r_{g}, 2084.733 r_{g}, 2917.95 r_{g}$ for Fig. $2 b$ respectively, which indicates that the outflow sonic point increases with a decrease in the adiabatic index of the outflow and thus the wind starts with a very low bulk velocity which is why the mass-loss rate decreases. It is also observed that the sonic point of the accretion-'self-wind' system is, in general, located closer to the event horizon compared to the outflow sonic point for all values of $\mathcal{E}, \dot{M}_{E d d}, \gamma_{\text {in }}$ and $\gamma_{o}$.

Combining the informations obtained from the Fig. 2a and $2 \mathrm{~b}$, one can observe that for a fixed set of values of

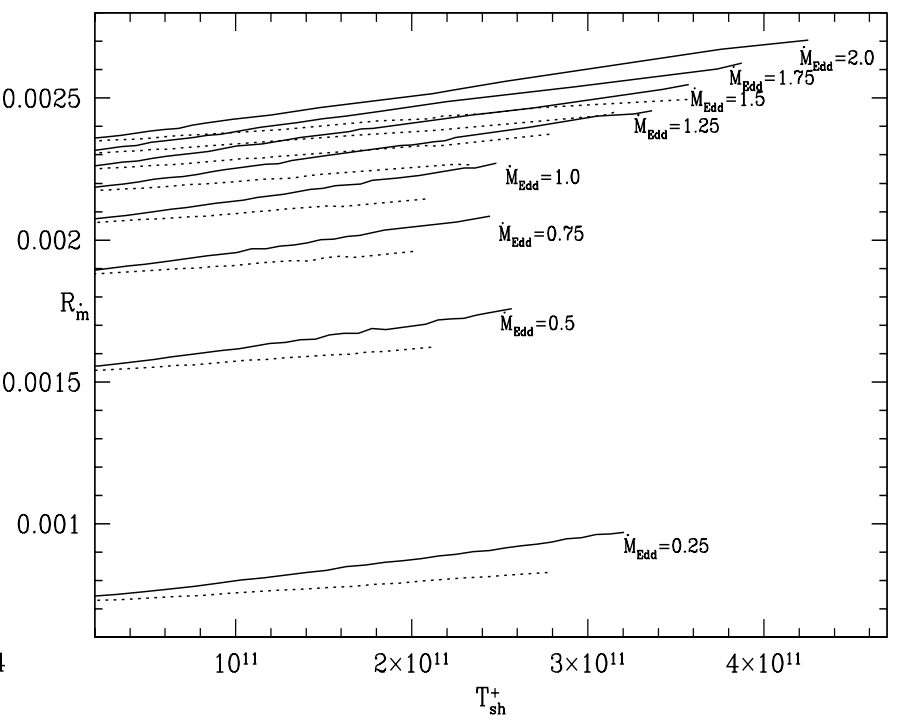

Fig. 3b: Variation of $R_{\dot{m}}$ with post-shock proton temperature $T_{s h}^{+}$corresponding to values of $\mathcal{E}$ shown in Fig. $3 \mathrm{a}$.

$\left\{\mathcal{E}, \dot{M}_{E d d}, \gamma_{i n}, \gamma_{o}\right\}$, the inflow sonic point (shown by $P_{s}$ in the figures) and the outflow sonic points (for various $\gamma_{o}$ ) for flows in $\Phi_{2}$, is greater than those quantities obtained for flow in $\Phi_{1}$. Whereas the shock Mach number $M_{s h}$ for $\Phi_{1}$ has a higher value compared to $M_{s h}$ for $\Phi_{2}$. This is obvious because the potential $\Phi_{2}$ is steeper compared to $\Phi_{1}$ (see DS) hence $\Phi_{2}$ produces relatively higher acceleration and the accreting material gains more kinetic energy relatively faster to become supersonic at a distance relatively greater compared to the flow in $\Phi_{1}$ and as the sonic point of the inflow resides relatively further away from the event horizon, accreting material becomes supersonic at an earlier stage and the rate of increment of the ratio of dynamical (mechanical) to the thermal energy content of the accretion increases; also another contributing factor is the location of the shock, Eq. (15) also tells that $M_{s h}^{+}$for $\Phi_{2}$, in reality, should be less compared to its value for $\Phi_{1}$. However, for any set of $\left\{\mathcal{E}, \dot{M}_{E d d}, \gamma_{i n}, \gamma_{o}\right\}$, the mass outflow rate $R_{\dot{m}}$ for $\Phi_{1}$ is less than that obtained in $\Phi_{2}$. We will come to this point in detail in next sub-section.

\subsection{Dependence of $R_{\dot{m}}$ on the specific energy of the flow}

In figure 3 a, we have plotted the variation of $R_{\dot{m}}$ with the specific energy of the flow $\mathcal{E}$ for a fixed values of $\gamma_{i n}\left(=\frac{4}{3}\right)$ and $\gamma_{o}(=1.3)$ and for a number of values (shown in the figure) of the accretion rate (scaled in units of Eddington rate and shown in the figure as $\dot{M}_{E d d}$ ) of the inflowing material for flows in $\Phi_{1}$ (dotted lines), as well as for flows in $\Phi_{2}$ (solid lines). Any dotted line accompanying a solid line marked by a specific value of $\dot{M}_{E d d}$ corresponds to the same accretion rate as that of the solid lines. We observe that for a fixed value of $\dot{M}_{E d d}, \gamma_{i n}$ and $\gamma_{o}$, the mass-outflow rate non-linearly correlates with $\mathcal{E}$ reason for which might be as follows:

As $\mathcal{E}$ increases, $r_{s h}$ decreases (see Fig. $1, \S 3.1$ ) and the post- 


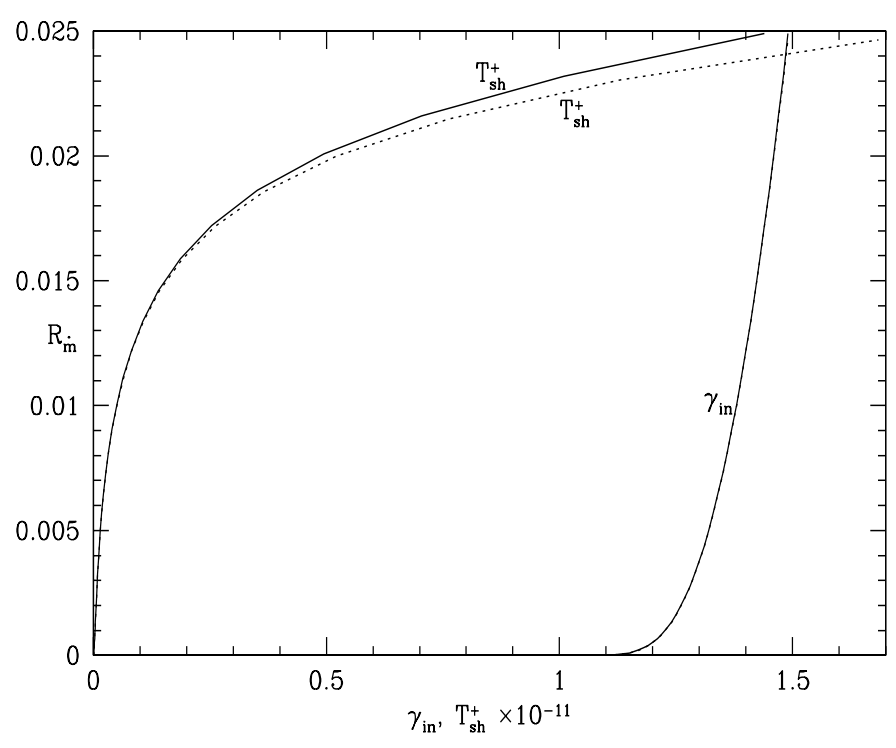

Fig. 4: Variation of $R_{\dot{m}}$ with the polytropic index of accretion $\gamma_{i n}$ (curves marked by $\gamma_{i n}$ ) and the corresponding post-shock temperature (curves marked by $T_{s h}^{+}$) for flows in $\Phi_{1}$ (dotted curve) and in $\Phi_{2}$ (solid curve). The Mass-outflow rate non-linearly correlates with $\gamma_{i n}$ as well as with post-shock proton temperature which indicates that the outflow is thermally driven as well, see text for details.

shock bulk velocity of the flow $u_{s h}^{+}$as well as the post-shock density $\rho_{s h}^{+}$increases. The outflow rate, which is the product of three quantities $r_{s h}, \rho_{s h}^{+}$and $u_{s h}^{+}$(see Eq. (11b)), increases in general due to the combined 'tug of war' of these three quantities. Moreover, the closer the shock is to the black hole, the greater the amount of gravitational potential will be available to be put onto the relativistic protons to provide stronger outward pressure and the closer the shock forms to the black hole, the higher is the post-shock proton temperature (the effective characteristic outflow temperature) and the higher is the amount of outflow (as the wind is observed to be strongly thermally driven, see discussion below). Thus the mass-outflow rate increases with $\mathcal{E}$ because for a particular set of fixed values of $\dot{M}_{E d d}, R_{\dot{m}}$ is proportional to $\dot{M}_{\text {out }}$ which increases with $\mathcal{E}$.

The unequal gaps between the curves marked with different $\dot{M}_{E d d}$ in the figure imply that when the inflow specific energy is kept constant, $R_{\dot{m}}$ non-linearly increases with the accretion rate of the infalling material. This is because, as $\mathcal{E}$ is kept constant while $\dot{M}_{E d d}$ is varied, the amount of infalling energy converted to produce the high-energy protons is also fixed. So the higher the value of $\dot{M}_{E d d}$, the larger the distance of the shock surface from the event horizon and the outflowing matter feels a low inward gravitational pull, the result of which is the non-linear correlation of $R_{\dot{m}}$ with $\dot{M}_{E d d}$. In other words, for a fixed value of $\gamma_{i n}$, high energy high luminosity accretion produces more outflow for both of the pseudo-potentials considered here. Also one can observe that for a fixed set of parameters $\left\{\mathcal{E}, \dot{M}_{E d d}, \gamma_{i n}, \gamma_{o}\right\}$, the amount of mass-outflow for $\Phi_{2}$ is higher compared to that in $\Phi_{1}$.

In figure $3 \mathrm{~b}$, we plot $R_{\dot{m}}$ as a function of $T_{s h}^{+}$for $\Phi_{1}$ (dotted line) and $\Phi_{2}$ (solid line) corresponding to the parameters used in the figure $3 \mathrm{a}$. We see that post-shock temperature non-linearly correlates with the energy of the flow, and for a fixed accretion rate and adiabatic indices of the inflow and outflow, $R_{\dot{m}}$ also correlates with post-shock temperature, which indicates that the outflow is thermally driven as well.

\subsection{Variation of $R_{\dot{m}}$ with adiabatic indices of the flow}

In previous cases, the polytropic index $\gamma_{i n}$ of the accreting matter was always kept fixed at the value $\frac{4}{3}$. To have a better insight of the behaviour of the outflow, we plot $R_{\dot{m}}$ as a function of $\gamma_{i n}$ (curves marked by $\gamma_{i n}$ in Fig. 4) for a fixed value of $\mathcal{E}(=0.00001)$ and $\dot{M}_{E d d}(=1.0)$. The upper range of $\gamma_{i n}$ shown here is the range for which shock forms for the specified value of $\mathcal{E}$ and $\dot{M}_{E d d}$. We have chosen the value of $\gamma_{o}$ in such a way so that the condition $\gamma_{o}<\gamma_{i n}$ is always satisfied. Defining $\Delta_{\gamma}$ to be $\Delta_{\gamma}=\gamma_{i n}-\gamma_{o}$, we study the variation of $R_{\dot{m}}$ with $\gamma_{i n}$ for $\Delta_{\gamma}=0.01$. One can also obtain results for other values of $\Delta_{\gamma}$ in the same way. We observe that $R_{\dot{m}}$ correlates with $\gamma_{i n}$, which is expected because the specific enthalpy of the flow increases with $\gamma_{i n}$ to produce a higher post-shock temperature for higher value of $\gamma_{i n}$ (see also the curves marked by $T_{s h}^{+}$in Fig. 4). We observe from our calculation that $\rho_{s h}^{+}$and $u_{s h}^{+}$correlates while $r_{s h}$ anticorrelates with $\gamma_{i n}$. So increment of $\gamma_{i n}$ satisfies all possible conditions to have a high value of $R_{\dot{m}}$. In the same figure, curves marked by $T_{s h}^{+}$show the variation of $R_{\dot{m}}$ with $T_{s h}^{+}$corresponding to the values of $\gamma_{i n}$ shown in the figure (scaled $T_{s h}^{+} \longrightarrow T_{s h}^{+} \times 10^{-11}$ to fit in the same figure) to show that here also the flow is thermally driven. Also, we observe that $M_{s h}^{+}$as well as the shock compression ratio $R_{c o m p}$ correlates with $\gamma_{\text {in }}$ so that 'strong-shock' solutions are preferred to obtain a high value of mass-loss for this case. So we conclude that as the accretion approaches from its ultra-relativistic nature to its non-relativistic regime, the mass-loss rate increases.

It is observed that for same set of parameters used to study the dependence of $R_{\dot{m}}$ on $\gamma_{i n}$, flow in $\Phi_{2}$ produces more mass-loss compared to flow in $\Phi_{1}$, i.e.,

$$
\left.R_{\dot{m}}\right|_{\Phi_{2}}>\left.R_{\dot{m}}\right|_{\Phi_{1}}
$$

for any value of $\gamma_{i n}$ and the quantity

$$
\delta R_{\dot{m}}=\left[\left.R_{\dot{m}}\right|_{\Phi_{2}}-\left.R_{\dot{m}}\right|_{\Phi_{1}}\right]
$$

decreases with decrease of $\gamma_{i n}$. However, in the figure, the curves showing the dependence of $R_{\dot{M}}$ with $\gamma_{i n}$ are not properly resolved to explicitly show the difference for flows in $\Phi_{1}$ and $\Phi_{2}$ due to the scaling used here to show both the temperature dependence of $R_{\dot{M}}$ as well as the dependence of $R_{\dot{M}}$ on $\gamma_{i n}$ in the same figure.

We also study the variation of $R_{\dot{M}}$ with $\gamma_{o}$ (figure not presented in this paper) for fixed values of $\left\{\mathcal{E}, \dot{M}_{E d d}, \gamma_{i n}\right\}$. The general conclusion is that $R_{\dot{m}}$ correlates with $\gamma_{o}$ for both of the pseudo-potentials. This is because as $\gamma_{o}$ increases, shock location and post-shock density of matter does not change 
(as $\gamma_{o}$ does not have any role in shock formation or in determining the $R_{\text {comp }}$ ) but the sonic point of the outflow is pushed inward, hence the velocity with which outflow leaves the shock surface here also we found that $R_{\dot{m}}$ for flow in $\Phi_{2}$ is always greater than than $R_{\dot{m}}$ for $\Phi_{1}$ and the deviation becomes more prominent as the value $\gamma_{o}$ approaches to $\gamma_{i n}$. goes up, resulting the increment in $R_{\dot{m}}$.

\section{CONCLUSION}

In this paper, we could successfully construct a selfconsistent spherically-symmetric, polytropic, transonic, non-magnetized inflow-outflow system by simultaneously solving the set of hydrodynamic equations governing the accretion and wind around a Schwarzschild black hole using two different pseudo-potentials proposed by Artemova et. al. (1996). Introducing a steady, standing, hadronic-pressure supported spherical shock (formation of which was first proposed by KE86 and PK83) surface around the black hole as the effective physical atmosphere which may be responsible for generation of accretion-powered spherical wind, we calculate the mass-outflow rate $R_{\dot{m}}$ in terms of only three accretion parameters (conserved energy of the flow $\mathcal{E}$, accretion rate $\dot{M}_{E d d}$ scaled in units of Eddington rate and polytropic index of the flow $\gamma_{i n}$ ) and only one outflow parameter (the polytropic index of the outflow, $\gamma_{o}$ ). Not only do we provide a sufficiently plausible estimation of $R_{\dot{m}}$, we could also successfully study the dependence and variation of this rate on various physical parameters governing the flow.

The basic conclusions of this paper may be summarized as:

(i) Shock formation is not a generic phenomena, i.e., not all solutions contain shock, rather a specific region of parameter space spanned by $\mathcal{E}, \dot{M}_{E d d}$ and $\gamma_{i n}$ allows shock formation. For given values of $\mathcal{E}, \dot{M}_{E d d}$ and $\gamma_{i n}$, while the value of shock location (in units of $r_{g}$ ) correlates with $\dot{M}_{E d d}$, it anti-correlates with both $\mathcal{E}$ and $\gamma_{i n}$. High energy high luminosity purely non-relativistic accretion is a proper choice to produce high post-shock proton temperature( thus to produce the maximum outflow). For other accretion parameters being the same, $\Phi_{1}$ produces a shock closer to the black hole compared to $\Phi_{2}$. This deviation is more prominent for high $\mathcal{E}$ and $\gamma_{\text {in }}$.

(ii) The shock surface can serve as the 'effective' physical barrier around the black hole regarding generation of mass loss via transonic spherical wind. The fraction of accreting material being blown as wind (which is denoted as $R_{\dot{m}}$ ) could be computed in terms of three accretion parameters and one outflow parameter.

(iii) $R_{\dot{m}}$ correlates with $\mathcal{E}, \dot{M}_{E d d}, \gamma_{i n}$ and $\gamma_{o}$, Outflow could be generated for both sub-Eddington as well as superEddington accretion. For a fixed set of $\left\{\mathcal{E}, \dot{M}_{E d d}, \gamma_{i n}, \gamma_{o}\right\}$, mass outflow rate $R_{\dot{m}}$ is higher for flows in $\Phi_{2}$ compared to flows in $\Phi_{1}$, which indicates that the barionic content of the spherical wind is inversely proportional to the spatial gradient on the pseudo-potential used to study the problem though the exact physical reason behind this is not quite clear.

(iv) If a shock forms, then whatever the initial flow conditions and whatever the nature of dependence of $R_{\dot{m}}$ on any of the accretion/ shock/ outflow parameters, $R_{\dot{m}}$ normally correlates with post-shock flow temperature, which indicates that outflow is strongly thermally driven; hotter flow always produces more winds.

At this point, it is worth mentioning that the hot and dense shock surface around black holes, which is proposed here as the effective physical barrier around compact objects regarding the mass outflow, may be generated due to other physical effects as well for spherical accretion (Chang \& Osttriker 1985, Mészáros \& Ostriker 1983, Babul, Ostriker \& Mészáros 1989, Park 1990, 1990a). Another very important approach launched recently was to construct such an 'effective barrier' for non-spherical disc accretion to introduce the concept of CENtrifugal pressure supported BOundary Layers (CENBOL). Treating the CENBOL as the effective atmosphere of the rotating flows around compact objects (which forms as a result of standing Rankine-Hugoniot shock or due to the maximization of polytropic pressure of accreting material in absence of shock), detailed computation of the massoutflow rate from the advective accretion disks has been done, and dependence of this rate on various accretion and shock parameters has been quantitatively studied by constructing a self-consistent disk-outflow system (Das 1998, Das \& Chakrabarti 1999).

Our calculations in this paper, being simply founded, do not explicitly include various radiation losses and cooling processes, combined effects of which may reduce the post-shock proton temperature (which means the reduction of outflow temperature), in reality could be lower than what we have obtained here and the amount of outflow would be less than what is obtained in our calculation. This deviation will be more important for systems with high accretion rates. Nevertheless, cases of low accretion rates discussed here would not be affected that much and our preliminary investigation shows that even if we incorporate various losses, the overall profile of the various curves showing the dependence of $R_{\dot{m}}$ on different inflow parameters would be exactly the same, only the numerical value of $R_{\dot{m}}$ in some cases (especially for high accretion) might decrease.

Although in this work we have performed our calculation for a $10 M_{\odot}$ Schwarzschild black hole, general flow characteristics will be unchanged for black hole of any mass except the fact that the region of parameter space responsible for shock formation will be shifted and the value of $R_{\dot{m}}$ will explicitly depend on the mass of the black hole.

\section{REFERENCES}

Artemova, I. V., Bjornsson, G., \& Novikov, I. D. 1996, ApJ, 461, 565

Babul, A., Ostriker, J. P., \& Mészáros, P. 1989, ApJ, 347, 59

Bondi, H. 1952, MNRAS, 112, 195

Chang, K. M., \& Ostriker, J. P. 1985, ApJ, 288, 428

Das, T. K. 1998, in Observational Evidence for Black Holes in the Universe, Ed. S. K. Chakrabarti (Kluwer Academic: Holland), p. 113

Das, T. K. 1999, MNRAS, 308, 201 (D99)

Das, T. K., \& Sarkar, A. 2001, A \& A, 374,1150 (DS)

Ellision, D. C., Eichler, D. 1984, ApJ, 286, 691

Ellision, D. C., Eichler, D. 1985, Phys. Rev. Letters, 55, 2735

Frank, J., King, A., \& Raine, D. 1992, Accretion Power in Astrophysics. 2nd. Edition. Cambridge University Press. 
Kazanas, D., \& Ellison, D. C. 1986, ApJ, 304, 178 (KE86)

Paczyński, B., \& Wiita, P. J. : 1980, A \& A, 88, 23

Park, M. G. 1990, ApJ, 354, 64

Park, M. G. 1990a, ApJ, 354, 83

Protheroe, R. J., \& Kazanas, D. 1983, ApJ, 256, 620 (PK83)

Tarafdar, S.P. 1988, ApJ, 331, 932 